\title{
Die Verhaltenstherapie als genuin psychologisch?
}

\author{
Zum Verhältnis zwischen Psychologie und Medizin \\ am Max-Planck-Institut für Psychiatrie in den 1960er \\ und 1970er Jahren
}

Lisa Malich

Universität zu Lübeck

Zusammenfassung. Die Klinische Psychologie ist heute von der Verhaltenstherapie geprägt. Eine Erklärung für diese enge Verbindung liefert die These, dass die Verhaltenstherapie ein genuin psychologisches Verfahren sei: Sie stamme historisch direkt aus der Disziplin Psychologie. Als eigenes Verfahren habe sie der Psychologie die Abgrenzung zur Medizin ermöglicht, die zuvor lange den Bereich der Psychotherapie dominiert habe. Im vorliegenden Artikel soll diese These für den bundesdeutschen Raum untersucht und das Verhältnis zwischen Verhaltenstherapie und Klinischer Psychologie genauer betrachtet werden. Dazu wird eine mikrohistorische Untersuchung vorgenommen, die sich auf den Zeitraum der 1960er und 1970er Jahre des Max-Planck-Instituts für Psychiatrie konzentriert. Dessen psychologische Abteilung unter Johannes Brengelmann gilt in vielen psychologiegeschichtlichen Darstellungen als grundlegend für die Entwicklung der Verhaltenstherapie ebenso wie der Klinischen Psychologie in Deutschland. Anhand von Archivmaterialien sowie Veröffentlichungen der psychologischen Abteilung rekonstruiere ich zwei verschiedene Figuren, mit denen zentrale Protagonist_innen die Beziehung der Verhaltenstherapie zur Psychologie charakterisierten: Zum einen erfolgte teilweise eine Abgrenzung zur Medizin und die Betonung einer eigenen Identität in der Psychologie. Zum anderen kam es immer wieder zu einer Annäherung der Verhaltenstherapie an die Medizin, zu der etwa die Positionierung als methodische Erneuerung der Psychiatrie, die Orientierung an medizinischen Modellen psychischer Erkrankungen und die Zusammenarbeit in der klinischen Praxis zählten. Insgesamt kann die untersuchte These für die deutsche Entwicklung in dieser Phase also nicht bestätigt werden. Vielmehr ist in Bezug auf Verhaltenstherapie von einem komplexen Verhältnis zwischen Psychologie und Medizin auszugehen, das zwischen Kooperation und Konkurrenz changierte.

Schlüsselwörter: Psychologiegeschichte, Wissenschaftsgeschichte, Klinische Psychologie, Psychiatrie

Behavior Therapy as Genuinely Psychological? On the Relationship Between Psychology and Medicine at the Max Planck Institute for Psychiatry in the 1960s and 1970s

Abstract. Clinical psychology today is characterized largely by behavioral therapy. One explanation for this close connection is the thesis that behavior therapy was historically the first therapeutic approach that came genuinely from the discipline of psychology. In this way, according to the thesis, psychology was able to draw boundaries between it and medicine, which had long dominated the field of psychotherapy. This article will examine this thesis for the Federal Republic of Germany and take a closer look at the relationship between behavior therapy and psychology. To this end, a microhistorical investigation was conducted, focusing on the period of the $1960 \mathrm{~s}$ and $1970 \mathrm{~s}$ at the Max Planck Institute for Psychiatry. In many psychological-historical accounts, the psychology department of the institute, which was headed by Johannes Brengelmann, is considered to be fundamental to the development of behavioral therapy as well as clinical psychology in Germany. Using archive material and publications from the psychology department, two different factors were found to be involved in reconstructed with which central protagonists characterized the relationship of behavioral therapy to psychology and medicine. On the one hand, there was a partial demarcation from medicine and an emphasis on psychology's identity on its own terms. On the other hand, behavioral therapy in that period was getting close to medicine in various ways. These included self-representation as a methodological renewal of psychiatry, an orientation toward medical models of mental illness, and cooperation in practice. Therefore, the thesis as examined could not be confirmed for German developments in this phase. Rather, a complex relationship between psychology and medicine must be assumed, which oscillated between cooperation and competition.

Keywords: psychology history, scientific history, clinical psychology, psychiatry

Im Jahr 2020 startete an deutschen Universitäten der neue Direktstudiengang Psychotherapie. Verortet ist der
Studiengang fast immer an psychologischen Instituten, wo der Master meist den Titel „MSc in Psychologie mit 
Schwerpunkt Klinische Psychologie und Psychotherapie“ trägt. Sowohl die institutionelle Zuständigkeit als auch die Namensgebung verdeutlichen, wie eng heute die Verbindung zwischen Psychotherapie und der Disziplin Psychologie ist, insbesondere in Bezug auf die Subdisziplin Klinische Psychologie. Zugleich beschäftigt sich die Klinische Psychologie nicht mit allen in Deutschland zugelassenen psychotherapeutischen Verfahren, sondern konzentriert sich auf Ansätze aus dem Feld der Verhaltenstherapie. So erklären Hans-Ulrich Wittchen und Jürgen Hoyer in ihrem einschlägigen Lehrbuch, allein schon wegen der begrifflichen Unschärfe von ,Psychotherapie' lasse ,sich fachintern die Konvention treffen, uns als Klinische Psychologen ausschließlich auf den besser definierten [...] Begriff einer weiter gefassten, Verhaltenstherapie zu beziehen" (Wittchen, 2011, S. 452). Gemäß dem Psychotherapieforscher Falk Leichsenring hatten 2018 insgesamt 59 der 60 Professuren für Klinische Psychologie in Deutschland eine kognitiv-behaviorale Ausrichtung (Bühring, 2018).

Die Zuständigkeit der Disziplin Psychologie für die Psychotherapie erscheint uns heute als selbstverständlich, ebenso wie die enge Verbindung zwischen Klinischer Psychologie und Verhaltenstherapie. Dennoch haben diese Verknüpfungen eine jeweils eigene Geschichte und sind das Ergebnis verschiedener, teilweise kontingenter historischer Prozesse.

Zunächst zum Zusammenhang zwischen der Disziplin Psychologie und Psychotherapie. Zwar entstanden beide Felder ungefähr zeitgleich, nämlich im ausgehenden 19. Jahrhundert - ansonsten hatten sie aber im deutschsprachigen Raum zunächst kaum Berührungspunkte. Führende Vertreter der akademischen Psychologie in Deutschland lehnten es ab, sich mit psychotherapeutischen Praktiken zu beschäftigen, die damals vor allem aus psychoanalytischen und individualpsychologischen Schulen kamen (Lück \& Guski-Leinwand, 2014). Dahingegen wurde Psychotherapie lange Zeit vorwiegend von Mediziner_innen ausgeübt, gelehrt und definiert (Roelcke, 2008). Erst nach einigen Jahrzehnten kam es zu einer losen Verbindung von akademischer Psychologie, psychopathologischer Diagnostik und Psychotherapie. So hatte die erste deutsche Diplomstudienordnung der Psychologie von 1941 Praktika in der Psychiatrie oder in psychotherapeutischen Beratungsstellen vorgesehen (s. Wieser, dieses Heft). Zudem gab es ab den 1950er Jahren in der Rahmenordnung Psychologie das Diplomprüfungsfach "Tiefenpsychologie und Psychagogik“" (Hoyos, 1964, S. 84; Schorr, 1990). Doch gerade im Vergleich zu den USA gewann die Disziplin Psychologie in Deutschland relativ spät (nämlich um fast fünf Jahrzehnte versetzt) an Bedeutung für die Psychotherapie. Während sich bereits 1919 eine Sektion Clinical Psychology in der American
Psychological Association gebildet hatte, fand die Sektionsgründung Klinische Psychologie im Berufsverband Deutscher Psychologen (BDP) in Westdeutschland erst 1963 statt (Vangermain \& Brauchle, 2010). Die akademische Entwicklung dauerte noch länger, 1984 kam es zur Gründung der Fachgruppe Klinische Psychologie in der Deutschen Gesellschaft für Psychologie (DGPs). Ausgangspunkt für diese Institutionalisierung in der BRD war die Verankerung des damals neuen Fachs ,Klinische Psychologie' in der reformierten Rahmenordnung des Diplomstudiengangs Psychologie im Jahr 1973. Denn das nun deutschlandweit vorgeschriebene Fach, das bei Studierenden sehr beliebt war, begünstigte das Einrichten von entsprechenden Professuren an allen psychologischen Instituten der Universitäten (Tändler, 2016). Die meisten dieser neu eingerichteten klinisch-psychologischen Lehrstühle wurden mit verhaltenstherapeutisch orientierten Wissenschaftler_innen besetzt (Daiminger, 2007).

Wie kam es dazu, dass die Klinische Psychologie in Deutschland so bald nach ihrer Einführung durch die Verhaltenstherapie geprägt war?

Auf die Frage nach der engen Verbindung zwischen Klinischer Psychologie und Verhaltenstherapie gibt es verschiedene gute Antworten (z. B. Vangermain \& Brauchle, 2010), von denen in diesem Aufsatz nur eine innerhalb der akademischen Psychologie verbreitete These näher herausgegriffen und diskutiert werden sollen. Diese bezieht sich auf die disziplinäre Entstehung der Verhaltenstherapie, nämlich darauf, dass diese der erste Therapieansatz gewesen sei, der, genuin psychologisch' war. So erklärt ein aktuelles Lehrbuch der Verhaltenstherapie: „Als genuin psychologischer Heilkundeansatz kann die Verhaltenstherapie mit besonderem Recht die Bezeichnung ,psychologische Psychotherapie" beanspruchen" (Schneider \& Margraf, 2019). Oft wird die These der genuin psychologischen Verhaltenstherapie auch auf ihre allgemeine Positionierung im Verhältnis zwischen den Disziplinen Psychologie und Medizin ausgeweitet: Während psychodynamische und tiefenpsychologische Ansätze vorwiegend in der Hand der Medizin gewesen seien, habe die Verhaltenstherapie zur Psychologie gehört. So ist etwa zu lesen, dass das „Konfliktpotenzial zwischen Psychoanalyse und Verhaltenstherapie“ auch auf die "Konkurrenz Medizin/Psychologie" zurückzuführen sei (Wittmann, 1981, S. 66). Vangermain und Brauchle erwähnen in ihrer facettenreichen Geschichte des Psychotherapeutengesetzes (s. dazu auch Schulte, dieses Heft) unter anderem die Erklärung, dass es sich bei der Verhaltenstherapie um ein Verfahren gehandelt habe, „das dem ärztlichen Behandlungsmonopol [...] genuin psychologische, empirisch wissenschaftliche [...] Behandlungskonzepte entgegensetzte" (2010, S. 96). Der Begriff des ,Genuinen' in der mehrfach genannten Charakterisierung eines, genuin psychologi- 
sches' Verfahrens impliziert die Idee der Abstammung und damit auch die einer historisch gedachten Herkunft. Die enge Orientierung der Klinischen Psychologie an der Verhaltenstherapie wird so über einen gemeinsamen disziplinären Ursprung und über die Abgrenzung zur Medizin begründet.

Tatsächlich sprechen viele Indizien für diese These, kamen doch Personen, die heute oft als zentrale Gründerfiguren der Verhaltenstherapie dargestellt werden, aus der Psychologie, allen voran John B. Watson mit seinem psychologisch-behavioristischen Manifest, ebenso wie Mary Cover Jones oder Hans Eysenck. Das betrifft besonders Protagonist_innen aus den USA oder Großbritannien, wo ein Studienabschluss in Psychologie früher möglich war als in Deutschland. Doch auch in Westdeutschland kamen einige Personen, die entscheidend zur Verbreitung der Verhaltenstherapie beitrugen, aus der dort damals noch jungen Diplompsychologie. Dabei handelte es sich etwa um die Psychologin Erna Duhm, Mitgründerin und erste Vorsitzende der klinischen Sektion im BDP, die eine der ersten Professuren für Klinische Psychologie in Göttingen erhielt (Viquerat, 2017). Häufig taucht auch der Name Johannes Brengelmann in diesem Kontext auf (Daiminger, 2007; Fichter, 2006; Vangermain \& Brauchle, 2010). Brengelmann hatte in Göttingen studiert und dort ein Psychologiediplom erhalten. Nach einem 10-jährigen Aufenthalt zunächst bei dem bekannten Psychologen Hans Eysenck und dann beim Psychiater Aubrey Lewis in London, wurde er 1965 der Leiter der psychologischen Abteilung am Max-Planck-Institut für Psychiatrie (MPIP) in München.

Zur These von der Nähe zwischen Verhaltenstherapie und Psychologie gehört mitunter noch ein Unteraspekt, der sich nicht auf die disziplinäre Herkunft, sondern auf konzeptionell-ätiologische Elemente bezieht. Denn manche Narrative führen aus, die Verhaltenstherapie stehe inhärent im Gegensatz zum ,medizinischen Modell' psychischer Beschwerden: Sie betone die Lernerfahrung und den Einfluss von Umweltreizen anstelle von körperlichen Faktoren wie genetischen oder neuronalen Dispositionen (Elberfeld, 2020). Dies war durchaus schon in der relativ frühen US-amerikanischen Verhaltenstherapie der Fall, deren prominenteste Figur B.F. Skinner sich ab den 1950er Jahren vom medizinischen Modell distanzierte und stattdessen die Bedeutung der Umwelt hervorhob (Goddard, 2014; Rutherford, 2009). Außerdem postulierten gerade in den 1970er und 1980er Jahren viele Vertreter_innen des Behaviorismus, dass der psychologische Fokus auf Lernprozesse in einem Spannungsverhältnis zu rein somatischen Erklärungsmodellen bzw. dem „physiologischen Paradigma" stehe (Erwin, 1978; Davison \& Neale, 1979, S. 50). Dazu zählten auch einige westdeutsche Verhaltenstherapeut_innen, vor allem mit ge- meindepsychologischer Ausrichtung, die in den 1970er Jahren in Konflikt mit medizinischen Krankheitsmodellen und psychiatrischen Methoden traten (Daiminger, 2007).

Dieser Aufsatz befasst sich mit der Geschichte des Verhältnisses zwischen Verhaltenstherapie und den Disziplinen Psychologie und Medizin in Deutschland. Dazu soll besonders die vorgestellte These vom genuin psychologischen Ursprung der Verhaltenstherapie kritisch beleuchtet werden. Fasste die Verhaltenstherapie hier tatsächlich Fuß, weil es sich um einen dezidiert ,psychologischen' Ansatz handelte, der sich sowohl disziplinär als auch konzeptionell klar von der Medizin unterschied? Hierbei konzentriert sich die Analyse nicht auf das gesamte Feld der Klinischen Psychologie und Verhaltenstherapie, sondern auf einen kleinen Ausschnitt - wenngleich auf einen zentralen: Auf die psychologische Abteilung des MPIP unter Johannes Brengelmann. In seiner Abteilung gründete sich 1968 die Gesellschaft zur Förderung der Verhaltenstherapie (GVT), die einer von mehreren Vorläufern der heutigen DGVT ist (Daiminger, 2007). 1971 formierte sich ebenfalls dort die European Association of Behavior Therapy mit Brengelmann als ihrem ersten Präsidenten. Viele Mitarbeiter_innen des Instituts wurden später zu wichtigen Figuren der Verhaltenstherapie und Klinischen Psychologie, etwa Roman Ferstl, Hans-Ulrich Wittchen, Kurt Hahlweg oder Renate de Jong-Meyer. Deswegen erklärt Jürgen Margraf in einem Zeitzeugeninterview, Brengelmann habe ,jede Menge Professoren produziert, ich glaube, mehr als jeder andere" (Daiminger, 2007, S. 135). Dies betraf besonders die in den 1970er Jahren neu eingerichteten klinisch-psychologischen Lehrstühle.

Die mikrohistorische Untersuchung bezieht sich auf den prägenden Zeitraum der 1960er bis 1970er Jahre, in dem nicht nur die psychologische Abteilung am MPIP gegründet wurde und sich vergrößerte, sondern sich auch generell in Westdeutschland maßgebliche disziplinäre und professionelle Weichenstellungen vollzogen. Die Klinische Psychologie institutionalisierte sich erfolgreich an den Universitäten, und die Verhaltenstherapie verbreitete sich in der Praxis. Das Quellenmaterial der Analyse basiert auf Institutsakten des Archivs der Max-Planck-Gesellschaft (AMPG) sowie auf akademischen Publikationen der psychologischen Abteilung des MPIP.

\section{Vorgeschichte: Das MPIP}

Das Institut, das später zum MPIP werden sollte, wurde 1917 als Deutsche Forschungsanstalt für Psychiatrie (DFA) in München vom Mediziner Emil Kraepelin gegründet. 1924 wurde die Anstalt in die renommierte und 
finanzkräftige Kaiser-Wilhelm-Gesellschaft eingegliedert (Weber, 1991). Bald stieg das Institut zu einer international führenden Forschungsstätte der somatischen Psychiatrie auf, also desjenigen Zweiges der Psychiatrie, der psychische Erkrankungen auf körperlich-biologische Ursachen zurückzuführen versucht (Harrington, 2019). Im Institut dominierte damit das, was heute als das medizinische Modell psychischer Erkrankungen bezeichnet wird. Auch allgemein war die akademische Psychiatrie in Deutschland zu Beginn des 20. Jahrhunderts von solch einem somatischen Zugang geprägt und grenzte sich vehement von den fast zeitgleich aufkommenden psychotherapeutischen Zugängen ab. Zwar praktizierten einige Mediziner_innen, unter ihnen auch Psychiater_innen, die neuen therapeutischen Methoden - von den zentralen Vertretern der Universitätspsychiatrie, so auch an der DFA, wurden diese aber als unwissenschaftlich weitgehend abgelehnt (Roelcke, 2008). Die Disziplin Medizin in Deutschland war damals also in mindestens zwei Lager gespalten: in die meist somatisch orientierte Psychiatrie und in psychotherapeutisch bzw. teilweise psychosomatisch orientierte Ärzt_innen. Als sich 1928 die ,Allgemeine ärztliche Gesellschaft für Psychotherapie' gründete, tat sie dies deswegen dezidiert in Abgrenzung zur Psychiatrie (Lockot, 1985). In dieser ersten deutschen psychotherapeutischen Gesellschaft dominierten psychodynamische Ansätze. Zugleich gab es dort aber auch Vertreter_innen symptombezogener Verfahren, wie dem autogenen Training, das in den 1920er Jahren vom Arzt Johannes Heinrich Schultz eingeführt worden war (Brunner \& Steger, 2006).

Als Institution der somatischen Psychiatrie wandte sich die DFA gegen jegliche psychotherapeutischen Praktiken und vereinte stattdessen verschiedene zeitgenössische Zugänge zu einer körperlichen Ätiologie von ,Geisteskrankheiten', die von chemischen und biologischen Ansätzen über pathologisch-anatomische Untersuchungen des Gehirns bis hin zu eugenischen Ansätzen reichten (Max-Planck-Gesellschaft, 1983). Kraepelin, der seinerseits auch Schüler Wilhelm Wundts war, richtete am Institut eine experimentell-psychologische Abteilung ein, die zur genaueren Erforschung hirnphysiologischer Prozesse dienen sollte. Hier wurden beispielweise toxikologische Versuche zu veränderten Reaktionszeiten nach dem Konsum von Alkohol durchgeführt. Doch in den Folgejahren setzte sich besonders die Eugenik am Institut durch - also die Lehre, die davon ausging, dass Geisteskrankheiten auf eine ,degenerative' Veranlagung zurückzuführen sei und deswegen dem ,Volkskörper' schadeten. Im Nationalsozialismus unterstützte Ernst Rüdin, Leiter der sogenannten ,genealogisch-demographischen Abteilung' der Anstalt aktiv die rassenhygienische Selektionspolitik von Zwangssterilisation und Euthanasie (Roelcke,
2002). In anderen Abteilungen nutzen einige Mitarbeiter_innen in dieser Zeit Gehirne, die aus Krankenmorden stammten, für ihre hirnpathologische Forschung (Steger, 2006).

Nach dem Ende des zweiten Weltkriegs waren Teile des Instituts zerstört und viele Abteilungen - so auch die experimentell-psychologische - nicht mehr vorhanden. Ernst Rüdin, der ab 1931 der geschäftsführende Direktor des gesamten Instituts gewesen war, wurde von der amerikanischen Militärregierung aller Ämter enthoben. Nach ihm übernahm 1945 der Mediziner Willibald Scholz die Geschäftsführung der Forschungsanstalt, die zu Beginn der 1950er Jahre formell in die Max-PlanckGesellschaft (MPG) - die Nachfolgegesellschaft der KWG - aufgenommen worden war. Er wurde 1961 von dem Neuropathologen Gerd Peters als Leiter abgelöst, der nach einer kurzen Phase, in der an der Anstalt psychotherapeutische (damals psychodynamische und verstehend-anthropologische) Ansätze integriert worden waren - eine klare Rückkehr zur somatischen Psychiatrie forderte. Unter Peters wurde die psychologische Abteilung eingerichtet und Brengelmann kam ans Institut. 1965, mit dem Startschuss Brengelmanns Abteilung, war der Aufstieg der Klinischen Psychologie und Verhaltenstherapie in der BRD eingeläutet. Schon durch die Bezeichnung ,psychologische Abteilung' scheint ihr psychologischer Charakter selbstverständlich - doch ist das tatsächlich der Fall? Und weshalb erfolgte die Gründung einer psychologischen Abteilung mit behavioraler Prägung genau dann, als sich die Leitung des MPIPs eigentlich auf die Tradition in der somatischen Psychiatrie besann?

\section{Ambivalente Kooperationen: Die Psychologie in der Psychiatrie}

Schon in der Gründungsphase der psychologischen Abteilung positionierten sich ihre Vertreter wieder in der Nähe zur Medizin. Dies wurde dadurch erleichtert, dass Brengelmann selbst nicht nur Psychologe, sondern auch Arzt war. 1944 hatte er sein medizinisches Staatsexamen in Göttingen abgelegt, 1945 seine medizinische Dissertation zu Amputationstechniken abgeschlossen, 1947 hatte er sein Diplom in Psychologie erhalten, 1949 wurde er mit einer Arbeit über den Aufbau des Gedächtnisses promoviert. Dass Psychologen in Deutschland zunächst Medizin oder ein anderes Fach wie Pädagogik studiert hatten, ist nicht unbedingt überraschend. Schließlich konnte man in Deutschland erst ab 1941, der Einführung der Studienordnung, einen Studienabschluss in Psychologie erhalten - Brengelmann gehörte in Deutschland also 
überhaupt zu den ersten offiziellen Diplompsychologen. Neben seiner Funktion als Psychologe betonte er in dieser frühen Phase allerdings besonders seine Verankerung in der Medizin, besonders der Psychiatrie.

Schon die Initiative zu einer psychologischen Abteilung ging von Brengelmann selbst aus. Bereits sieben Jahre vor ihrer eigentlichen Gründung, nämlich im Jahr 1957, hatte er einen Brief an den damaligen Generalsekretär der MPG geschrieben, in dem er diesen auf die problematische Situation der deutschen Psychiatrie und Psychopathologie - und nicht der Psychologie - aufmerksam machen wollte, die im internationalen Vergleich stark abgefallen sei (Dok. 1). In dem Schreiben führte er aus, die zeitgenössischen „deutschen philosophisch orientierten Methoden“ verwiesen nur mehr „in die Geschichte der Psychiatrie“. Damit spielte er auf die traditionelle Spaltung in der deutschen Medizin an, die sich nun in der Nachkriegszeit fortsetzte: Einerseits kam es in der Psychiatrie weiten Teilen zu einer Rückkehr zu somatischen Modellen, die gleichzeitig mit einer Modernisierung und dem Einbezug experimenteller und statistischer Methoden einherging. Andererseits institutionalisierte sich nun der konkurrierende psychotherapeutische Zugang in psychosomatischen Kliniken (Roelcke, 2012). Letzteres begann im Jahr 1949/50, in dem der Mediziner Alexander Mitscherlich gegen den Widerstand der dortigen Psychiatrie die erste Abteilung für ,allgemeine Therapie' an der klinischen Universitätsanstalt Heidelberg gründete (Geisthövel \& Hitzer, 2019). Die Psychosomatik war meist psychodynamisch geprägt und zeichnete sich in den 1950er Jahren zudem durch eine philosophische Wende hin zu Neukantianismus und Daseinsanalyse aus (Bohleber, 2010). In seinem Brief richtete sich Brengelmann also gegen diesen, philosophisch orientierten' Ansatz, dafür sah er in der Rückkehr zum Experiment die mögliche Erneuerung der Psychiatrie. Zu diesem Zweck schlug er der MPG das Einrichten einer experimentalpsychologischen Abteilung „nach britischen oder amerikanischen Muster" vor. Zum Leiter solch einer Abteilung „müsste ein Experimentalpsychologe benannt werden, der medizinisch wie psychologisch ausgebildet ist" - nämlich er selbst.

Unterstützt wurde Brengelmanns Anliegen einen Monat später durch ein weiteres Schreiben an die MPG. Es stammte von Johannes von Allesch, Professor für Psychologie an der Universität Göttingen und damals Vorsitzender der DGPs (Dok. 2). Darin lobte von Allesch Brengelmann in höchsten Tönen: „In Deutschland ist kein Psychologe, der zugleich Mediziner ist, zu finden, der [...] für die in Frage kommende Stellung auch nur annähernd so geeignet erscheint." In dem mehrseitigen Schreiben ist nur diese eine Zeile unterstrichen - wahrscheinlich vom Empfänger in der MPG, für den offensichtlich Brengelmanns gleichzeitige Verankerung in der Medizin zentral war. Als in einer Sitzung der MPG im Jahr 1964 schließlich der Vorschlag, eine psychologische Abteilung einzurichten und Brengelmann als Leiter aufzunehmen, diskutiert wurde, begründete man dies mit den „Aufgaben der zukünftigen psychiatrischen Klinik“, zu denen der „Aufbau einer experimentellen Psychopathologie" gehöre (Dok. 3). Um die Nutzung der Abteilung für die psychiatrische Forschung nicht zu gefährden, wurde damals beschlossen, diese als unselbständige Abteilung mit weniger Autonomie einzurichten, die dem klinisch-psychiatrischen Institutsteil unterstellt wurde und Forschung für diesen übernehmen sollte. In diesem Kontext schien die Psychologie nicht als autonome Disziplin, sondern als methodisch-experimenteller Zugang, der von der Psychiatrie genutzt wurde. Aus Sicht der Psychiatrie sollte sie also in erster Linie als Instrument einer Verwissenschaftlichung der somatischen Psychiatrie nach dem Zweiten Weltkrieg dienen.

Die Betonung von Brengelmanns Doppelqualifikation und die Unterordnung der Psychologie unter die Psychiatrie mag teilweise strategisch gewesen sein, um auf die umfangreichen Ressourcen der Psychiatrie am MPIP zugreifen zu können. Dazu kamen aber noch inhaltliche und epistemologische Überschneidungen zu zeitgenössischen psychiatrisch-medizinischen Ansätzen. Brengelmann beschäftigte sich vor allem mit Persönlichkeitspsychologie, dem Einfluss von Lernen auf Leistung und der Entwicklung standardisierter Tests. In der bekannten Reihe „Psychiatrie der Gegenwart" hatte er 1963 ein langes Kapitel zu „Psychologischer Methodik und Psychiatrie“ veröffentlicht. Zuvor hatte er eine englischsprachige Monographie zum Effekt von Elektroschockbehandlungen beim Lernen von Menschen mit Depressionen publiziert und sich danach stärker der Psychopharmakologie als neuem, ab den 1950er Jahren allgemein aufstrebenden Gebiet in der Psychiatrie zugewendet (Brengelmann, 1958, 1959; Brengelmann, Pare \& Sandler, 1959). Dazu gehörten Versuche mit Stoffen wie Amphetaminen, Amobarbital und LSD. Hierbei versuchte Brengelmann, eine differenzielle Psychopharmakologie einzuführen, die die Abhängigkeit von Substanzwirkungen je nach Persönlichkeitsmerkmal postulierte. Der Hintergrund Brengelmanns psychopharmakologischer Experimente war ein genereller Trend in dersomatischen Psychiatrie: Während zuvor viele in Deutschland führende Neurologen und Psychiater versucht hatten, der Ursache des ,Wahns' durch neurologische und hirnphysiologische Untersuchungen näher zu kommen, kam es in den 1950er Jahren zu einer psychochemischen Wende, die durch Experimente mit psychedelischen Stoffen wie LSD sowie durch die Einführung der ersten Psychopharmaka eingeläutet wurde (Tornay, 2016). Teile von Brengelmanns Forschung bezogen sich also explizit auf die Psychiatrie und orientierten sich an 
Grundannahmen eines medizinischen Modells psychischer Erkrankungen.

\section{Das medizinische Modell in der Verhaltenstherapie}

Doch betraf Brengelmanns Nähe zum medizinischen Modell auch seine Zugänge zu Behaviorismus und Verhaltenstherapie? Hierzu bietet sich ein Blick in seine Habilitationsschrift Bedingte Reaktionen, Lerntheorien und Psychiatrie von 1967 an, mit der er die Lehrerlaubnis für das Fach ,Klinische Psychologie' an der medizinischen Fakultät der Universität Düsseldorf erwarb. Darin entwirft er eine scharfe Opposition zwischen europäischen und US-amerikanischen behavioristischen Ansätzen der Lerntheorie. Während die ,amerikanische Richtung" die Persönlichkeit nur als „Summe aller erlernten Reaktionen“ verstehe und „mit allgemeinpsychologischen, universal gültigen Gesetzen“ arbeite, folge er der „europäischen Tradition", die individuelle Differenzen, erbliche Konstitution und Persönlichkeitstypen stärker betone (Brengelmann, 1967, S. 1041-1042). Darunter subsumiert er Pawlows Konditionierungsansatz, Kretschmers Konstitutionstypologie, charakterologische Ansätze und Eysencks Persönlichkeitsdimensionen. Die anschließende Darstellung beginnt Brengelmann mit einer Analyse des bedingten Reflexes nach Pawlows klassischem HundeExperiment - allerdings erklärt er dabei weniger die generellen psychologischen Prinzipien des klassischen Konditionierens, die heute damit verbunden werden. Vielmehr stellt er vor allem die Differenzen zwischen den Hunden heraus, die er auf körperliche Faktoren zurückführt. So gebe es Unterschiede in den ,nervösen Prozessen' bei der klassischen Konditionierung, die Pawlow in vier Typen eingeteilt habe: ,sanguinisch, lebhaft', ,melancholisch, schwach', ,cholerisch, ungestüm' und ,phlegmatisch, ruhig' (S. 1053). Von den differenzialpsychologischen Zugängen zum Lernen ausgehend, entwirft er auch eine Ätiologie der Psychopathologie: „Die Ausbildung abnormer Verhaltensweisen hängt von der Vererbung und der Umwelt $a b$ ", wobei besonders "genetische Faktoren eine bedeutsame Rolle" spielten (S. 1042). Insgesamt vertrat Brengelmann mit seinem betont, europäischen Ansatz also gerade nicht eine rein auf die Umwelt fokussierte Verhaltenstherapie. Vielmehr hob seine lerntheoretische Persönlichkeitspsychologie gerade differenzielle Konstitutionen und Vererbung hervor. Entsprechend anschlussfähig waren seine Ansätze an somatisch-psychiatrische Modelle, die biologische Ätiologien betonen - etwa Ansätze aus der Genetik und Hirnforschung.
Auch eine ausschließliche Verankerung in der Disziplin Psychologie ist in einer der ersten deutschsprachigen Publikationen zu Verhaltenstherapie nicht erkennbar. Es handelt sich um den von Brengelmann und Wolfgang Tunner herausgegebenen Sammelband Behavior Therapy - Verhaltenstherapie, der aus dem ersten Kongress der Europäischen Gesellschaft für Verhaltenstherapie entstand, der zugleich das dritte Treffen der deutschen Gesellschaft war. Im Vorwort charakterisieren die beiden Herausgeber die Verhaltenstherapie nicht als Unterbereich der Psychologie, sondern als eigene, übergeordnete ,junge Wissenschaft" beziehungsweise als „Disziplin [...], in der zum Zwecke therapeutischer Veränderung experimentelle Bedingungen auf der Basis der theoretischen und methodischen Erkenntnisse der Verhaltenswissenschaften, insbesondere der Lernpsychologie, eingesetzt werden" (Brengelmann \& Tunner, 1973, S. VI). Damit wird die Lernpsychologie hier zwar genannt, allerdings nur als ein Aspekt neben den breiteren Verhaltenswissenschaften. Noch weniger scheint Fritz Pirkl, der Bayerische Staatsminister für Arbeit und Sozialordnung, und selbst Diplompsychologe, in seinem Grußwort zum Kongress die Verhaltenstherapie als rein psychologisch zu betrachten. Hier hebt er immer wieder die Bedeutung von „Psychiatrie und Psychologie“ hervor (Brengelmann \& Tunner, 1973, S. 1), beziehungsweise diejenige von "Psychotherapie und Psychiatrie" (S. 2) und beschreibt die „Verhaltenstherapie als interdisziplinäre Aufgabe [...] von Medizin und Psychologie“ (S. 3). Entsprechend kommen zwar die meisten Beiträge des Sammelbands von Personen aus der Psychologie, einige Aufsätze stammen aber auch von Psychiater_innen oder Sozialarbeiter_innen. Statt als rein psychologisches Unterfangen erschient die deutschsprachige und europäische Verhaltenstherapie hier also früh als interdisziplinär.

Vor diesem Hintergrund ist wohl nicht verwunderlich, dass Mediziner_innen auf institutioneller und berufspraktischer Ebene bereits in der frühen Phase der Verhaltenstherapie involviert waren. International stammten einige frühe Vertreter der Lerntheorie wie Iwan Pawlow oder Joseph Wolpe aus der Medizin. In Westdeutschland zeigt sich die Nähe zwischen Verhaltenstherapie und Medizin etwa in der Voraussetzung für die Vollmitgliedschaft in der von Brengelmann gegründeten GVT, die gemäß Satzung ein abgeschlossenes Studium der Psychologie oder der Medizin erforderte. Für einige deutsche Mediziner_innen, insbesondere für Psychiater_innen, war die Verhaltenstherapie schon früh attraktiv. Denn in der traditionellen innermedizinischen Frontstellung zwischen somatischer Psychiatrie und ärztlicher Psychotherapie schien nun die neue Verhaltenstherapie eine Alternative zu bieten: als experimentell fundierter Ansatz, der von außen vor allem aus den USA und Großbritannien - kam, ließ 
sich diese Form der Psychotherapie leichter in die Psychiatrie integrieren. Zusätzlich trug zur Attraktivität der Verhaltenstherapie wohl auch ihre klare und verständliche Sprache bei, die im Gegensatz zu oft hermetisch wirkenden Ansätzen aus der zeitgenössischen Psychodynamik stand. Ein Zeitzeuge fasst die frühe Anziehung der Verhaltenstherapie knapp zusammen: „(...) von den Medizinern her: ,Endlich mal Psychotherapie, die man versteht."“ (Daiminger, 2007, S. 97). Als das MPIP ab 1966 eine eigene Klinik in München erhielt, arbeiteten und forschten dort Mediziner_innen und Psycholog_innen oft gemeinsam - und wendeten das neue verhaltenstherapeutische Verfahren so auch in der Praxis an.

Es gab also gerade in derjenigen Strömung der deutschen Verhaltenstherapie, die als besonders ,erfolgreich und prägend gilt, eine große Nähe zur Psychiatrie und zum medizinischen Modell. Die Integration von Psychologie und Verhaltenstherapie war aus mindestens zwei Gründen attraktiv für die Psychiatrie in Deutschland: Erstens wurden experimentalpsychologische Methoden häufig als Instrument zur Erneuerung und Modernisierung der Psychiatrie in Stellung gebracht. Zweitens bot die Verhaltenstherapie im lang schwelenden innermedizinischen Konflikt zwischen Psychiatrie und ärztlicher Psychotherapie für viele psychotherapeutisch interessierte Psychiater einen neutralen Ausweg, da sie experimentell ausgerichtet war und somatische Krankheitsmodelle aufgriff. Doch auch für Verhaltenstherapie und Psychologie war die Kooperation mit der Medizin von Vorteil, handelte es sich doch beim MPIP um eine finanzkräftige wie prestigereiche Institution mit eigener Klinik und zahlreichen Stellen für wissenschaftliche Mitarbeiter_innen. Die ausgezeichnete Ausstattung Brengelmanns psychologischer Abteilung und die ökonomischen Ressourcen des MPIP, die auch die Einladung internationaler Wissenschaftler ermöglichten, werden von Zeitzeugen immer wieder hervorgehoben (Daiminger, 2007). Es lässt sich somit vermuten, dass die Verwobenheit mit der Psychiatrie auch ein Grund für den Erfolg der Brengelmannschen Richtung der Verhaltenstherapie war, die ab den 1970er Jahren die meisten Professuren für Klinische Psychologie im westdeutschen Raum bestimmen sollte.

\section{Konkurrenzverhältnisse: Die Emanzipation der Psychologie von der Medizin}

Es würde jedoch zu kurz greifen, die Entwicklung der Verhaltenstherapie in der BRD allein aus ihrer Beziehung zur Disziplin Medizin, insbesondere zur Fachrichtung Psychia- trie, zu erklären. Die historischen Narrative, die die enge Verknüpfung zwischen Verhaltenstherapie und der Disziplin Psychologie betonen, treffen in Teilen durchaus zu.

Denn in disziplinärer Sicht bestand zwischen der Medizin und der aufstrebenden Psychologie auch Konkurrenz, was sich ebenfalls in Archivmaterialien des MPIP spiegelt. So ging es in den Beschlüssen um die Gründung der psychologischen Abteilung zwar nicht nur, aber eben auch um die Zukunft der Disziplin Psychologie. Dies betraf besonders die Interventionen von Alleschs, der ab 1957 bis zur Gründung der psychologischen Abteilung am MPIP mehrere Briefe an die Generalverwaltung der MPG schrieb. In den Briefen setzte sich von Allesch immer wieder vehement für seinen Schüler Brengelmann ein, vor allem aber für eine Integration der Disziplin Psychologie in die MPG. So betont von Allesch in einem Schreiben von 1957, dass die psychologische Abteilung auch „für die deutsche Psychologie im Ganzen, bei der gegenwärtig erneut eine Krisis der Methode droht, von großem Wert" sei (Dok. 2). Im Zuge der Diskussionen um die Einrichtung einer psychologischen Abteilung, die im Jahr 1964 erfolgte, spielten auch Argumente für die Stärkung der bundesdeutschen Psychologie insgesamt eine Rolle. Diese, so ein Sprecher, habe zu lange die „Rolle der Magd“ eingenommen, die dem „Psychiater Hilfsdienste bei der Diagnosestellung und gelegentlich auch in der Therapie" leiste - und sie müsse sich nun von der Psychiatrie emanzipieren (Dok. 3). In der Sitzung wurde zudem auf eine eben erschienene Denkschrift verwiesen, nämlich die von der DFG beauftragte Schrift zur Lage der Psychologie in Deutschland (Hoyos, 1964). Diese dokumentierte das massive Wachstum des Fachs Psychologie im Nachkriegsdeutschland. Zwischen 1950 und 1961 hatte sich die Zahl der Psychologiestudierenden in der BRD mehr als verdoppelt, von rund 739 Hauptfach-Studierenden im Jahr 1950/1951 hin zu ca. 1797 im Jahr 1960/61 (Hoyos, 1964, S. 95). Durch den Aufstieg des Fachs kam es zu inhaltlichen Diskussionen über die Ausrichtung und methodische Basis der psychologischen Forschung. Auch deswegen mahnte die Denkschrift das „Fehlen eines hochschulunabhängigen Instituts“ an, ,das sich ausschließlich mit der psychologischen Grundlagenforschung befaßt" und nannte hierbei explizit die MPG als Adressat (S. 27). Zwar entsprach die Einrichtung einer abhängigen psychologischen Abteilung an einem psychiatrischen Institut nicht unbedingt den Forderungen der Denkschrift - dass sie aber überhaupt in der Diskussion der MPG aufgenommen wurde, zeigt, dass die Abteilung durchaus als Teil der Disziplin Psychologie wahrgenommen wurde.

Dazu kam die personelle Seite: Die psychologische Abteilung unter Brengelmann stellte zunehmend Mitarbeiter_innen an, die einen Abschluss in Psychologie - nicht 
in Medizin - hatten. Diese waren teilweise auch in der Klinik des MPIP tätig, ebenso wie in verhaltenstherapeutischen Projekten eines privaten Forschungsinstituts, das Brengelmann 1973 in München gründete, das Institut für psychotherapeutische Forschung (IFT). Im Zuge davon veränderte sich der Tätigkeits- und Kompetenzbereich von Psycholog_innen in der Klinik. Schon zuvor waren Personen mit Psychologiestudium in psychiatrischen Kliniken tätig, allerdings in untergeordneter Rolle. Bisweilen polemisch als „Rechenknechte“ oder "Testdackel“ verunglimpft, übernahmen sie diagnostische Hilfstätigkeiten und durften nicht therapeutisch tätig sein (Daiminger, 2007, S. 72; Krampen, Miller \& Montada, 2002). Daneben konnten Psycholog_innen psychotherapeutisch arbeiten, wenn sie nach dem Studium eine mehrjährige Weiterbildung an außeruniversitären Instituten absolviert hatten - allerdings bevorzugten die psychodynamisch ausgerichteten Institute meist Mediziner_innen, die bei einer vorgegebenen Quote von ca. zwei Dritteln die Mehrheit bildeten. Dagegen bestand in der verhaltenstherapeutischen Ausbildung keine Quotierung für Psycholog_innen. Neben anderen Meilensteinen, die zum Aufstieg der psychologischen Psychotherapie beitrugen (Malich, 2020; Vangermain \& Brauchle, 2010), förderte die psychologische Abteilung am MPIP so durchaus die Integration der Psychologie in die psychotherapeutische Versorgung und Forschung. Auf diesem Wege emanzipierte sich die Psychologie von der Medizin insofern, als sich Psycholog_innen von fremdbestimmten ,Testdackeln ${ }^{6}$ $\mathrm{zu}$ eigenständig behandelnden psychologischen Psychotherapeut_innen und Klinischen Psycholog_innen entwickeln konnten.

Zugleich war die Situation am Münchner MPIP sehr spezifisch und kann, trotz ihres zentralen Einflusses auf die deutsche Psychologie und Psychotherapie, nicht verallgemeinert werden. Denn es gab in Westdeutschland noch andere frühere Hotspots der Verhaltenstherapie in den späten 1960er Jahren, die stärker psychologisch geprägt waren - so etwa in Göttingen, Münster oder Bochum (Daiminger, 2007). Außerdem kam es gerade zu Beginn der 1970er Jahre in der schnell wachsenden Verhaltenstherapie zu Richtungsauseinandersetzungen, bei denen sich gerade politisch engagierte Studierende der Psychologie und jüngere Psychotherapeut_innen von Brengelmann und dem medizinischen Modell distanzierten. Und nicht zuletzt unterschied sich die Situation der akademischen Psychologie und der Verhaltenstherapie in der BRD deutlich von derjenigen in anderen Ländern. Insbesondere in den USA, die für den damaligen bundesdeutschen Kontext prägend waren, hatte die Psychologie als eigenständige akademische Disziplin eine längere Tradition, so dass hier oft eine stärkere und institutionalisiere Abgrenzung zwischen der Medizin mit der Nähe zur Psy- choanalyse und der Klinischen Psychologie mit Nähe zum Behaviorismus bestand. $\mathrm{Zu}$ beachten ist dabei etwa, dass Psycholog_innen in den USA als nicht-ärztliche, Laien ' bis 1988 überhaupt nicht zur Ausbildung an psychoanalytisch Instituten zugelassen wurden (Norcross, VandenBos \& Freedheim, 2011), was sich von der deutschen Quotierung unterscheidet, durch die Psycholog_innen zumindest die Möglichkeit zur psychotherapeutischen Ausbildung hatten. Auf Grund der in den USA fester etablierten Trennung zwischen Medizin, Psychologie und den unterschiedlichen psychotherapeutischen Schulen entwickelte sich dort eventuell das Narrativ einer, genuin psychologischen Verhaltenstherapie', welches dann in Deutschland übernommen wurde. Insgesamt sollten weder , die Verhaltenstherapie‘ noch die ,Klinische Psychologie' monolithisch betrachtet werden - sie hatten stets nationale und lokale Dynamiken.

Abschließend lässt sich festhalten, dass die Verhaltenstherapie im deutschen Raum nicht alleine aus der Disziplin Psychologie hervorging. Für die psychologische Abteilung unter Johannes Brengelmann, die für die Verhaltenstherapie im deutschen Raum prägend war und deren Schüler_innen viele der ersten Professuren für Klinische Psychologie erhielten, lässt sich das historische Narrativ der genuin psychologischen Verhaltenstherapie, die sich klar von der Medizin abgrenzte, nicht halten. Vielmehr gestaltete sich das Verhältnis zur Klinischen Psychologie und Medizin ambivalenter - und changierte zwischen Konkurrenz und Kooperation.

\section{Archivalien}

Dokument 1: Brief vom 20.06.1957, Brengelmann an Ballreich, AMPG, II. Abt., Rep. 67, Nr. 392, fol. 305-308. Dokument 2: Brief vom 20.07.1957, von Allesch an Ballreich, AMPG, II. Abt., Rep. 67, Nr. 392, fol. 303-304.

Dokument 3: Sitzung der biologisch-medizinischen Sektion am 12.03.1964, AMPG, II. Abt., Rep. 67, Nr. 392, fol. 473.

\section{Literatur}

Bohleber, W. (2010). Psychoanalyse, Diktatur, Professionalität Implikationen: Die Auswirkungen des Nationalsozialismus auf die Psychoanalyse in der Bundesrepublik Deutschland nach 1945. In M. Ash (Hrsg.), Psychoanalyse in totalitären und autoritären Regimen (S. 293 - 315). Frankfurt a. M.: Brandes \& Apsel.

Brengelmann, J. C. (1958). D-amphetamine and amytal I: Effects on memory and expressive movement. Journal of Mental Science, 104, 153-159. 
Brengelmann, J. C. (1959). The Effect of Repeated Electroshock on Learning in Depressives. Berlin: Springer.

Brengelmann, J. C., Pare, C. M. B. \& Sandler, M. (1959). Effects of 5-hydroxytryptophan on schizophrenia. Journal of Mental Science, 105, $770-776$.

Brengelmann, J. C. (1963). Psychologische Methodik und Psychiatrie. In H. W. Gruhle, R. Jung, W. Mayer-Gross \& M. Müller (Eds.), Psychiatrie der Gegenwart (S. 134-175). Berlin: Springer-Verlag.

Brengelmann, J. C. (1967). Bedingte Reaktionen, Lerntheorien und Psychiatrie. München: Max-Planck-Institut für Psychiatrie.

Brengelmann, J. C. \& Tunner, W. (Eds.). (1973). Behaviour Therapy - Verhaltenstherapie. München: Urban \& Schwarzenberg.

Brunner, J. \& Steger, F. (2006). Johannes Heinrich Schultz (18841970) - Begründer des Autogenen Trainings. BIOS, 19 (1), $16-25$.

Bühring, P. (2018). Interview mit Prof. Dr. rer. nat. Falk Leichsenring: „Wir brauchen eine Vielfalt an evidenzbasierter Psychotherapie“. Deutsches Ärzteblatt, 449-450.

Daiminger, C. (2007). Eine Erfolgsgeschichte mit Differenzen: Zur Geschichte der Professionalisierung der Verhaltenstherapie und der Deutschen Gesellschaft für Verhaltenstherapie (DGVT) in der Bundesrepublik Deutschland. Tübingen: DGVT.

Davison, G. C. \& Neale, J. M. (1979). Klinische Psychologie: Ein Lehrbuch. München: Urban \& Schwarzenberg.

Elberfeld, J. (2020). Ambivalenzen der Kritik: Familientherapie und die Debatte um Schizophrenie in den 1970er Jahren. In V. Balz \& L. Malich (Eds.), Psychologie und Kritik: Formen der Psychologisierung nach 1945 (S. 187 -219). Berlin: Springer.

Erwin, E. (1978). Behavior Therapy: Scientific, Philosophical and Moral Foundations. London: Cambridge University Press.

Fichter, M. (2006). Hommage an Johannes C. Brengelmann (19201999) - drei seiner Schüler über die Aufbruchstimmung der frühen 1970er Jahre. Verhaltenstherapie, 16, 59-64.

Geisthövel, A. \& Hitzer, B. (2019). Auf der Suche nach einer anderen Medizin: Psychosomatik im 20. Jahrhundert. Frankfurt am Main: Suhrkamp.

Goddard, M. (2014). Critical Psychiatry, Critical Psychology, and the Behaviorism of B. F. Skinner. Review of General Psychology, 18, $208-215$

Harrington, A. (2019). Mind Fixers: Psychiatry's Troubled Search for the Biology of Mental Illness. New York, London: WW Norton \& Co.

Hoyos, C. G. (1964). Denkschrift zur Lage der Psychologie. Wiesbaden: Franz Steiner Verlag.

Krampen, G., Miller, M. \& Montada, L. (2002). Bibliometrische Untersuchungsbefunde zur Geschichte der Klinischen Psychologie im 20. Jahrhundert. Zeitschrift für Klinische Psychologie und Psychotherapie, 31, 121-126.

Lück, H. E. \& Guski-Leinwand, S. (2014). Geschichte der Psychologie: Strömungen, Schulen, Entwicklungen. Stuttgart: Kohlhammer.

Lockot, R. (1985). Erinnern und Durcharbeiten: Zur Geschichte der Psychoanalyse und Psychotherapie im Nationalsozialismus. Frankfurt a. M.: Fischer.

Malich, L. (2020). The History of Psychological Psychotherapy in Germany: The Rise of Psychology in Mental Health Care and the Emergence of Clinical Psychology during the 20th century. In W. Pickren (Ed.), Oxford Research Encyclopedia of Psychology. New
York: Oxford University Press. https://doi.org/10.1093/acre fore/9780190236557.013.628

Max-Planck-Gesellschaft. (Hrsg.). (1983). Max-Planck-Institut für Psychiatrie, München, Berichte und Mitteilungen 2/83. München: MPG.

Norcross, J. C., VandenBos, G. R. \& Freedheim, D. K. (Eds.). (2011). History of Psychotherapy: Continuity and Change. Washington, DC: American Psychological Association.

Roelcke, V. (2002). Programm und Praxis der psychiatrischen Genetik an der ,Deutschen Forschungsanstalt für Psychiatrie' unter Ernst Rüdin. Medizinhistorisches Journal, 37 (1), 21 - 55.

Roelcke, V. (2008). Rivalisierende „Verwissenschaftlichungen des Sozialen": Psychiatrie, Psychologie und Psychotherapie im 20. Jahrhundert. In R. J \& R. V (Hrsg.), Wissenschaften im 20. Jahrhundert (S. $131-148)$. Stuttgart: Steiner.

Roelcke, V. (2012). Psychotherapie in Westdeutschland nach 1945. Psychotherapeut, 2, 103-112.

Rutherford, A. (2009). Beyond the box: B. F. Skinner's technology of behavior from laboratory to life, 1950s-1970s. Toronto: University of Toronto Press.

Schneider, S. \& Margraf, J. (2019). Lehrbuch der Verhaltenstherapie (Bd. 3). Berlin: Springer.

Schorr, A. (1990). Verschenkter Neubeginn - Zur Tätigkeit beratender und behandelnder Psychologen im Nationalsozialismus und ihren Wirkungen in der Gegenwart. In A. Schorr \& E. G. Wehner (Hrsg.), Psychologiegeschichte heute (S. 239-260). Göttingen: Hogrefe.

Steger, F. (2006). Neuropathological Research at the ,Deutsche Forschungsanstalt fuer Psychiatrie' in Munich: Scientific Utilization of Children's Organs from the ,Kinderfachabteilungen“ at Bavarian State Hospitals. Journal of the History of the Neurosciences, 15, 173-185.

Tändler, M. (2016). Das therapeutische Jahrzehnt. Der Psychoboom in den siebziger Jahren. Göttingen: Wallstein.

Tornay, M. (2016). Zugriffe auf das Ich. Psychoaktive Stoffe und Personenkonzepte in der Schweiz, 1945 bis 1980. Tübingen: Mohr Siebeck.

Vangermain, D. \& Brauchle, G. (2010). Ein langer Weg der Professionalisierung: Die Geschichte des deutschen Psychotherapeutengesetzes. Verhaltenstherapie, 20, 93-100.

Viquerat, H. (2017). Nachruf auf die Grande Dame der Klinischen Psychologie. Zeitschrift für Klinische Psychologie und Psychotherapie, 46 (2), $139-142$.

Weber, M. (1991). „Ein Forschungsinstitut für Psychiatrie...“: Die Entwicklung der DFA in München zwischen 1917 und 1945. Sudhoffs Archiv, 75 (1), 74-89.

Wittchen, H.-U. \& Hoyer, J. (Eds.). (2011). Klinische Psychologie \& Psychotherapie. Heidelberg: Springer Medizin Verlag.

Wittmann, L. (1981). Verhaltenstherapie und Psychodynamik: therapeutisches Handeln jenseits der Schulengrenzen. Weinheim: Beltz.

\section{J.Prof. Dr. Dipl-Psych. Lisa Malich}

Institut für Medizingeschichte und Wissenschaftsforschung Universität zu Lübeck

Königstrasse 42

23552 Lübeck

lisa.malich@uni-luebeck.de 